\title{
Effect of Mode-Stirrer Configurations on Dielectric Heating Performance in Multimode Microwave Applicators
}

\author{
Pedro Plaza-González, Juan Monzó-Cabrera, José M. Catalá-Civera, Member, IEEE, and \\ David Sánchez-Hernández, Member, IEEE
}

\begin{abstract}
In this paper, several mode-stirrer configurations are compared in order to establish their influence on the electric-field uniformity within an irradiated dielectric sample inserted in a microwave-heating applicator. Two different scenarios are evaluated with metallic sheets moving inside the multimode applicator. The different stirrer configurations are tested and compared for low-, medium-, and high-loss dielectric sample materials. Additionally, a straightforward procedure based on a generalized plane-wave approach is proposed and evaluated as a computationally efficient alternative for calculating the electric-field distribution inside materials processed in these microwave applicators with mode stirrers. Although very different electric patterns are achieved depending on stirrer geometry and sample permittivity, the plane-wave approach has been shown to provide a very good approximation for medium and high lossy dielectric materials.
\end{abstract}

Index Terms-Dielectric materials, electromagnetic heating, finite-element analysis, mode stirrers, plane waves.

\section{INTRODUCTION}

$\mathbf{M}$ ODAL stirrers are the most widely accepted method to improve the uniformity of heating patterns on dielectric materials processed in multimode applicators, thus avoiding undesirable "hot spots." In a previous paper [1], a two-dimensional (2-D) method for inferring the electric-field distribution inside materials irradiated in multimode cavities with some mode stirrers was described and compared with classical approaches such as Lambert's Law [2]-[4]. Some simulation results obtained with the method described in [1], and validated with temperature measurements, stated that Lambert's Law could only be applied with acceptable accuracy to high loss dielectrics.

Despite the mode stirrers' movement, numerical and experimental results indicated that, for high-loss materials, a more intense electric field was found at the edges of the sample, which heated at a much higher rate than its center. Additionally, the

Manuscript received July 29, 2004; revised October 28, 2004. This work was supported in part by the Spanish Science and Technology Ministry under Project TIC2001-2778-CO2-02.

P. Plaza-González is with the Advance Communications and Information Technologies Research Institute, Universidad Politécnica de Valencia, 46022 Valencia, Spain (e-mail: pedplago@ doctor.upv.es).

J. Monzó Cabrera and D. Sánchez-Hernández are with the Departmento de Tecnologías de la Información y las Comunicaciones, Universidad Politécnica de Cartagena, 30202 Cartagena, Spain (e-mail: juan.monzo@upct.es; david.sanchez@upct.es).

J. M. Catalá-Civera is with the Departamento de Comunicaciones, Universidad Politécnica de Valencia, 46022 Valencia, Spain (e-mail: jmcatala@dcom.upv.es).

Digital Object Identifier 10.1109/TMTT.2005.847066 comparison of a constant electric-field distribution within the sample, which is commonplace in microwave-assisted drying processes [5], to the patterns obtained in [1] provided large discrepancies. This reduced further the range of validity for this constant distribution down to very thin materials. Simulated results in [1] also showed that both the permittivity and geometry of the sample had a strong influence on the field spatial distribution.

In this paper, different mode-stirrer configurations are explored in order to study their effect upon the sample electricfield distribution and to look for alternatives that avoid edge overheating in all kind of dielectric materials. Due to the fact that the possibilities for the mode stirrers' position, shape, and movement are very wide, only some of them have been investigated. Both the geometry and permittivity of the sample are again considered as the main parameters for this study.

Furthermore, a generalized plane-wave approach (GPWA) [6] is proposed here as an alternative to Lambert's Law to compare the calculation of the electric-field deposition within dielectric samples taking into consideration the reflections and stationary waves at the material-air interface. The differences between both approaches and the range of validity for the GPWA are discussed.

\section{NUMERICAL FORMULATION}

\section{A. Finite-Element Method (FEM) Approach Applied to the Multimode Cavity}

The multimode microwave applicators considered here are metallic cubic boxes that have been discretized as 2-D rectangular enclosures excited by the $\mathrm{TE}_{10}$ mode with a standard WR-340 waveguide, centered at the top of the cavity (see Fig. 1). These chambers contain mode stirrers inside with a continuous movement that enforces time-changing boundary conditions for the electric-field distribution.

The first example depicted in Fig. 1(a) consist of two vertical metallic plates both moving synchronously along the horizontal direction ( $x$-axis). Several positions of the moving metallic sheets ( $y$-axis) can be examined in order to verify the effect of the stirrer proximity to the sample. The second stirrer arrangement is represented by two metallic blades tracing a synchronous angular movement, as described in [1], but here, the possibility of different length is considered to evaluate new influences on the electric-field distribution within the sample. 


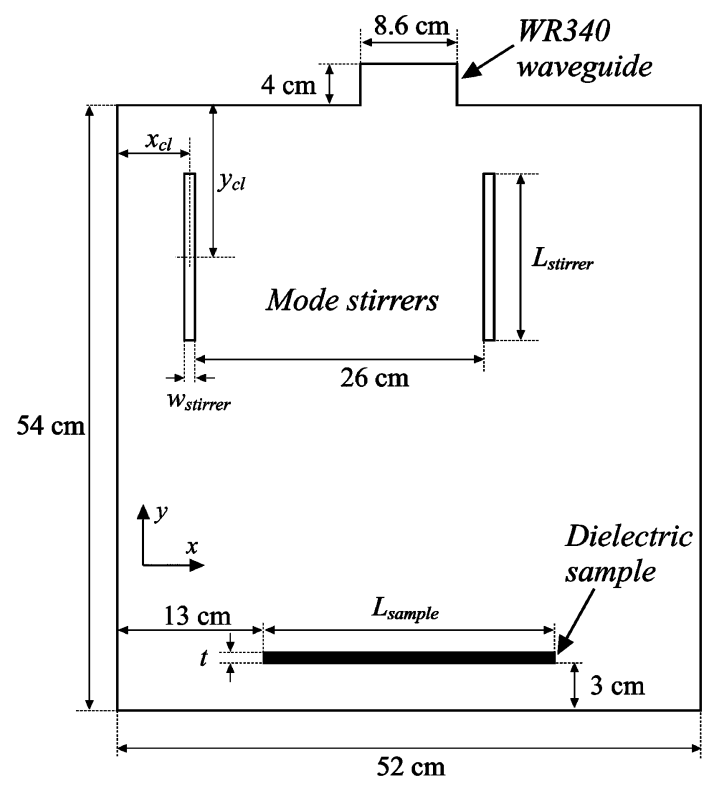

(a)

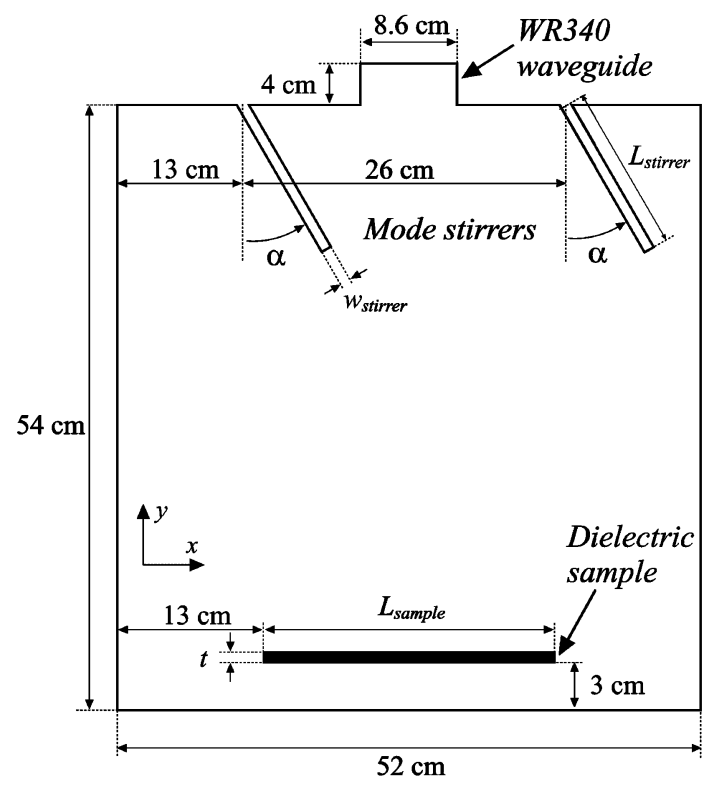

(b)

Fig. 1. 2-D simulation scheme for the multimode microwave cavity with two mode-stirrer configurations. (a) Horizontal and (b) angular movement.

The use of a 2-D approach versus the three-dimensional (3-D) is justified by the validation shown in [1] and the required high computing times, even for 2-D scenarios, to achieve a good precision for the electric field calculated within a large multimode cavity.

The numerical computation of the electric field within the multimode cavity and material is calculated for each arrangement by a quadratic average of $N$ contribution of the stirrers due to their movement, according to the procedure described in [1]. The Pdetool $^{1}$ function included in MATLAB was employed in order to apply the FEM procedure to a mesh in the 2-D domain.

${ }^{1}$ Partial Differential Equation Toolbox, MATLAB, Natick, MA. [Online]. Available: http://www.mathworks.com

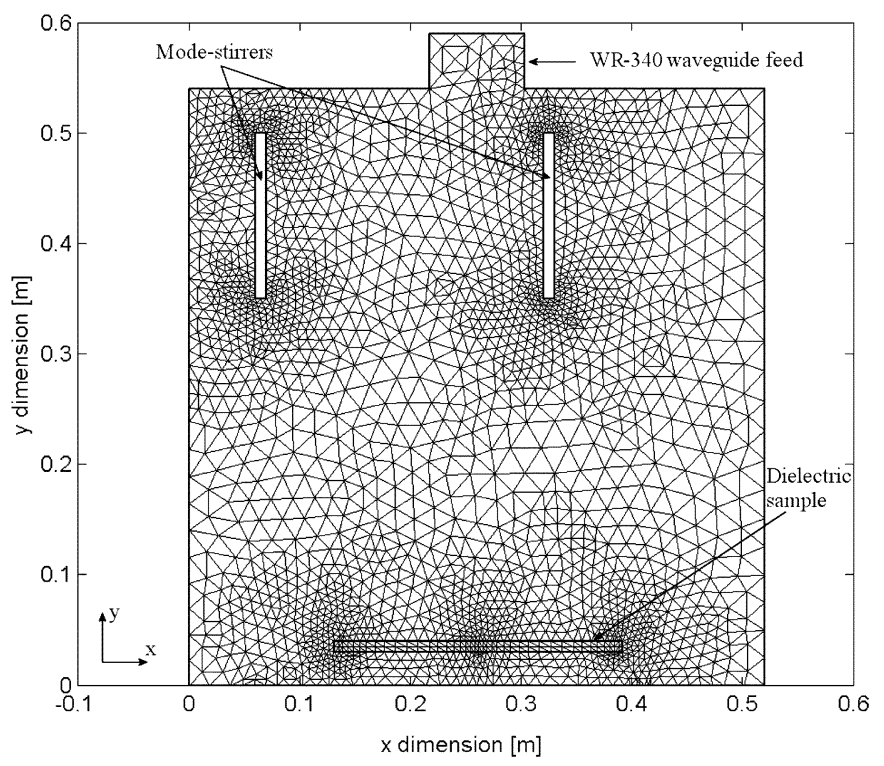

Fig. 2. 2-D simulation scheme for the multimode microwave cavity with mode stirrers and the associated mesh.

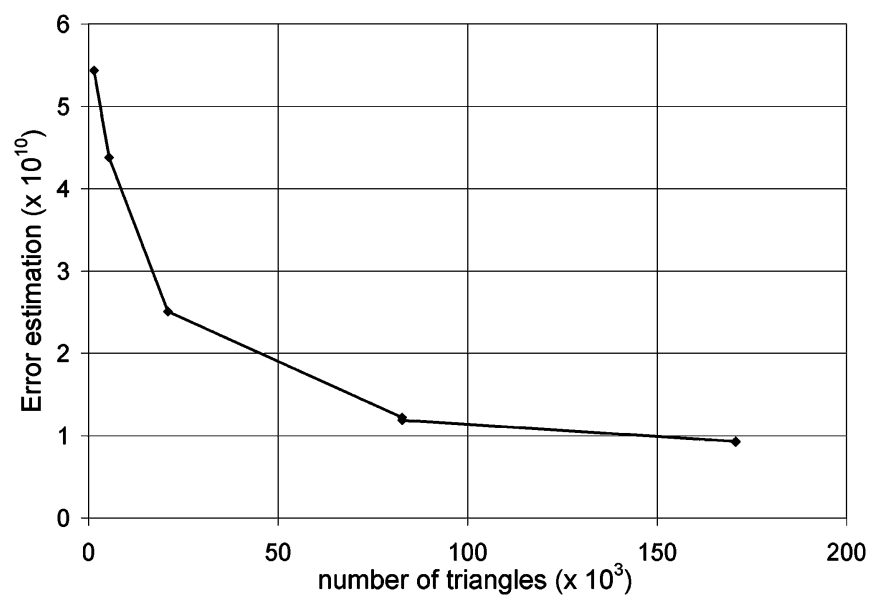

Fig. 3. FEM error estimation versus number of triangles in the mesh.

Fig. 2 shows an example of the adaptive mesh provided by the Pdetool function for an specific stirrers' configuration of the first arrangement examined here.

In order to obtain a precise solution with the FEM approach and to minimize computing times, a convergence study has been carried out. Equation (1), representing an error indicator, has been implemented and computed as follows for several triangle meshing refinement levels and the simulation scheme depicted in Fig. 2:

$E(k)=\alpha\|h(f-a u)\|_{k}+\beta\left(\frac{1}{2} \sum_{\tau \in \partial k} h_{\tau}^{2}\left[\vec{n}_{\tau} \cdot\left(c \nabla u_{h}\right)\right]^{2}\right)^{1 / 2}$

with $K$ being the triangle number, $h(f-a u)$ being the error at triangle $K, h_{\tau}$ being the length of the $\tau$ th side, and $\vec{n}_{\tau} \cdot\left(c \nabla u_{h}\right)$ being the change in normal derivative over side $\tau$. Both $\alpha$ and $\beta$ were set to 0.5 [7]-[8].

Fig. 3 shows this error indicator versus the number of meshing triangles. As can be observed, the error diminishes 


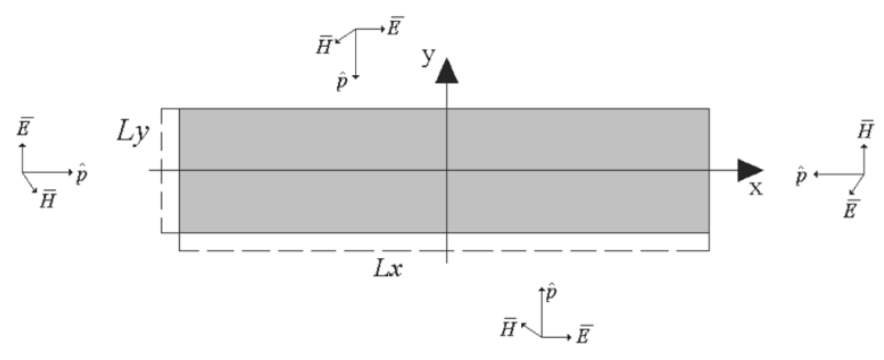

Fig. 4. Simulation scheme for the GPWA.

exponentially with increasing number of triangles. Yet computing time dramatically increases with the number of nodes. The use of a three-times refined mesh, which is equivalent to 82684 triangles, provided a good error indicator value. This configuration also provided acceptable computing times for all the simulation scenarios and, consequently, was selected as the meshing strategy.

\section{B. GPWA}

The dielectric material, as represented in Fig. 4, is assumed here as an homogeneous dielectric material of rectangular section in free space.

This dielectric, which represents the heated sample in the multimode cavity, is excited by an enforced field $E_{i}$ as combination of TM-polarized plane waves [6]

$$
E_{i}=\sum_{p} A_{p} e^{-j \vec{k}_{p} \cdot \vec{r}}
$$

$E_{i}$ excites polarization currents $J$ in the dielectric region, which produces a scattered field $E_{s}$. This field is related to the currents by the integral operator

$$
E_{s}=-\frac{k \eta}{4} \iint_{S} J \cdot H_{0}^{(2)}\left(k\left|\vec{r}-\vec{r}^{\prime}\right|\right) d S^{\prime}
$$

where $\eta=\sqrt{\mu / \varepsilon} \approx 120 \pi$ is the intrinsic impedance of free space and $H_{0}^{(2)}$ is the Hankel function of the second kind, zero order. Equation (3) is solved here by the method of moments (MoM) [9] by expanding the currents $J$ by base and weight functions

$$
J=\sum_{n=1}^{N} I_{n} J_{n} .
$$

A simplification of the electric field computed from previous equations is precisely the Lambert's Law since it does not consider the reflections at the dielectric-air interface. Assuming the dielectric material of Fig. 4 and four different planes waves propagating toward the center of the material, from different orthogonal angles, Lambert's Law is given by

$$
\begin{aligned}
& \left|\vec{E}_{\text {Lambert }}\right| \\
& \quad=\left|\vec{E}_{\text {surface }}\right|\left(e^{-\alpha x}+e^{-\alpha\left(x-L_{x}\right)}+e^{-\alpha y}+e^{-\alpha\left(y-L_{y}\right)}\right)
\end{aligned}
$$

where $\alpha$ is the attenuation factor $(N p / m)$, which depends upon the permittivity of the material [2], and $L_{x}$ and $L_{y}$ are the sample length in the canonical directions $x$ and $y$, respectively.
TABLE I

PARAMETERS AND GEOMETRY USED FOR FIGS. 5-8

\begin{tabular}{lll}
\hline \multirow{2}{*}{ Symbol } & QUANTITY & Value \\
\hline$f$ & Frequency & $2.45 \mathrm{GHz}$ \\
$E_{\text {source }}$ & TE $_{10}$ Electric field peak value & $5 \times 10^{3} \mathrm{~V} / \mathrm{m}$ \\
$\varepsilon^{\prime}$ & Sample dielectric constant & $2.94,15,40$ \\
$\varepsilon^{\prime \prime}$ & Sample loss factor & $0.2,2,12$ \\
$x_{c l}$ & Stirrer $1 x$ position & $0.035 \mathrm{~m}$ to $0.125 \mathrm{~m}$ in 32 \\
& & steps. \\
$y_{c l}$ & Stirrer $y$ sheet position & $0.105 \mathrm{~m} ; 0.245 \mathrm{~m} ; 0.385 \mathrm{~m}$ \\
$L_{\text {sample }}$ & Sample length & $0.26 \mathrm{~m}, \mathrm{x} \in[0.13,0.39] \mathrm{m}$ \\
$t$ & Sample thickness & $10 \mathrm{~mm}$ \\
& & $y_{\text {ini }}=0.03 \mathrm{~m}$ \\
& & $\mathrm{y}_{\text {end }}=0.04 \mathrm{~m}$ \\
& Multimode cavity dimensions & $x=0.52 \mathrm{~m}, y=0.54 \mathrm{~m}$ \\
& Stirrer dimensions & $x=0.01 \mathrm{~m}, y=0.15 \mathrm{~m}$ \\
\hline
\end{tabular}

In (2), the weight coefficients $A_{p}$ are calculated from the electric-field magnitude that best fit the quadratic minimum error for the behavior described by the FEM approach.

\section{NUMERICAL RESULTS AND DisCUSSION}

\section{A. FEM Simulations of the Multimode Cavity With} Mode Stirrers

The two different scenarios illustrated in Fig. 1 are assessed here. For the mode stirrer's configuration illustrated in Fig. 1(a), three vertical positions of the metallic plates are examined: one far from the material sample, (named Position I), another centered along the $y$-axis of the cavity (Position II), and a third one close to the dielectric load (Position III).

In the second arrangement, the metallic blades with angular movement of Fig. 1(b) are evaluated with three different lengths.

The permittivity of dielectrics for the simulations were selected from [2] taking into account values of real materials commonly processed with microwaves. These materials are $\varepsilon_{1}^{*}=$ $2.94-0.2 j$ ("Royal Grey" paper), $\varepsilon_{2}^{*}=15-2 j$ (30\% wet ceramic brick), and $\varepsilon_{3}^{*}=40-12 j$ (beef steak).

For each arrangement analyzed, the average electric-field distribution in the sample was computed for $N=32$ different stirrer's movements along the $x$-axis.

1) Scenario I: Vertical Stirrers With Horizontal Movement: The stirrer arrangement analyzed first corresponds to the schematic of Fig. 1(a) with the metallic plates at the top of the multimode cavity (Position I). Table I gives the geometric and physical description of the stirrers and materials.

Fig. 5 shows the electric-field distribution for four specific positions of the stirrers. As expected, we can observe in Fig. 5 very different electric-field distributions inside the cavity and sample, enforced by the horizontal movement of the metallic sheets.

Fig. 6 shows the average electric field at the center of the three dielectric materials for Position I of these stirrers, where we can appreciate a different number of stationary waves for each material. Likewise, the magnitude of the electric field decreasing as the complex permittivity increases, mainly due to the reflection created at the air-material discontinuity. For moderate- and high-loss materials, we can also observe the overexcitation at 

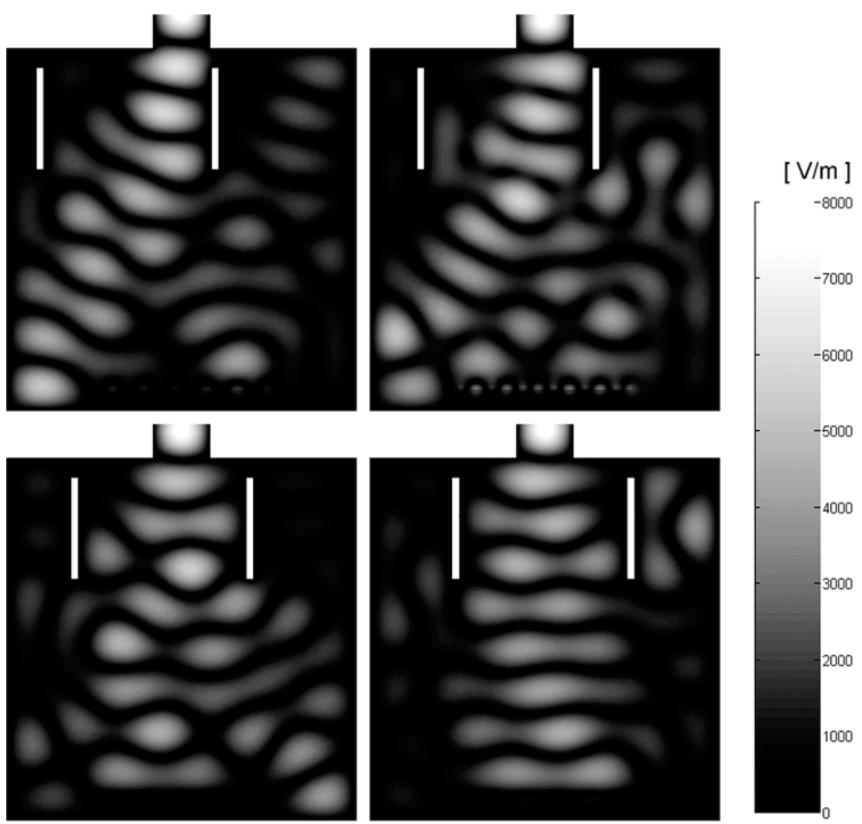

Fig. 5. Electric-field distribution (absolute value) in the multimode cavity for four different mode-stirrer positions $\left(x_{c l}=0.045,0.070,0.096\right.$ and $\left.0.122 \mathrm{~m}\right)$. $\varepsilon^{\prime}=40, \varepsilon^{\prime \prime}=12$, and material thickness $t=10 \mathrm{~mm}$.

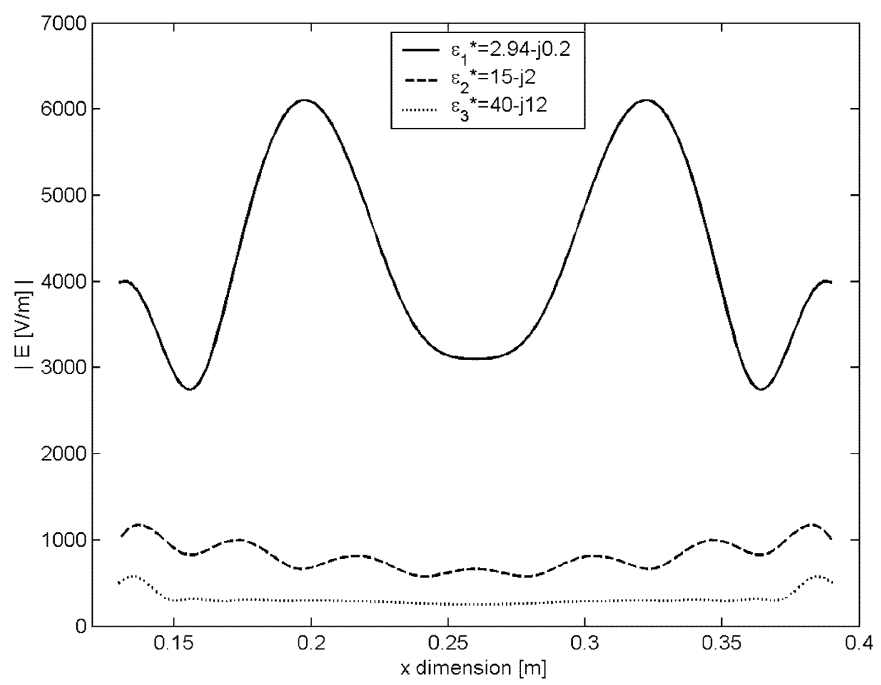

Fig. 6. Average electric-field distributions at the material center as a function of the sample permittivity. $t=10 \mathrm{~mm}$.

the edges, which is in accordance to the results reported in other stirrer configuration [1].

From the point-of-view of field uniformity, the results obtained for this new stirrer are similar to those obtained with previous designs. In order to assess this field uniformity with other stirrer configurations, the results showed in Fig. 6 are compared with the results obtained when shifting the stirrers to Positions II and III.

Fig. 7 shows the average electric field at the center of the three dielectric materials for stirrers placed at Positions I-III.

In these simulations, the electric-field pattern for the lower permittivity material is more uniform for the stirrer at Position III (lower ratio between the maximum and minimum magnitude of the electric field). From this comparison, we can conclude that the stirrer configuration is of the utmost importance in order

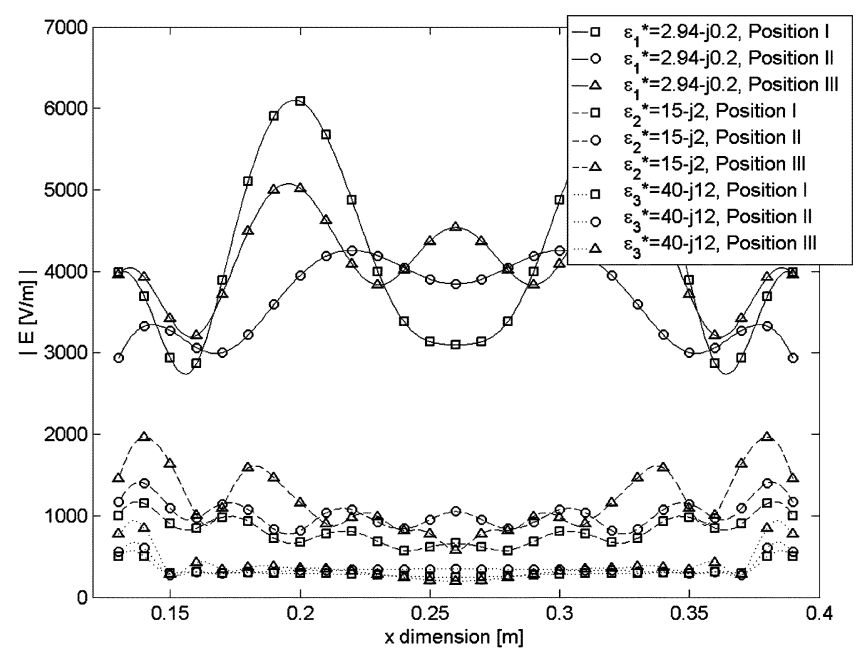

Fig. 7. Average electric-field distributions at the material center as a function of sample permittivity and stirrer position. $t=10 \mathrm{~mm}$.

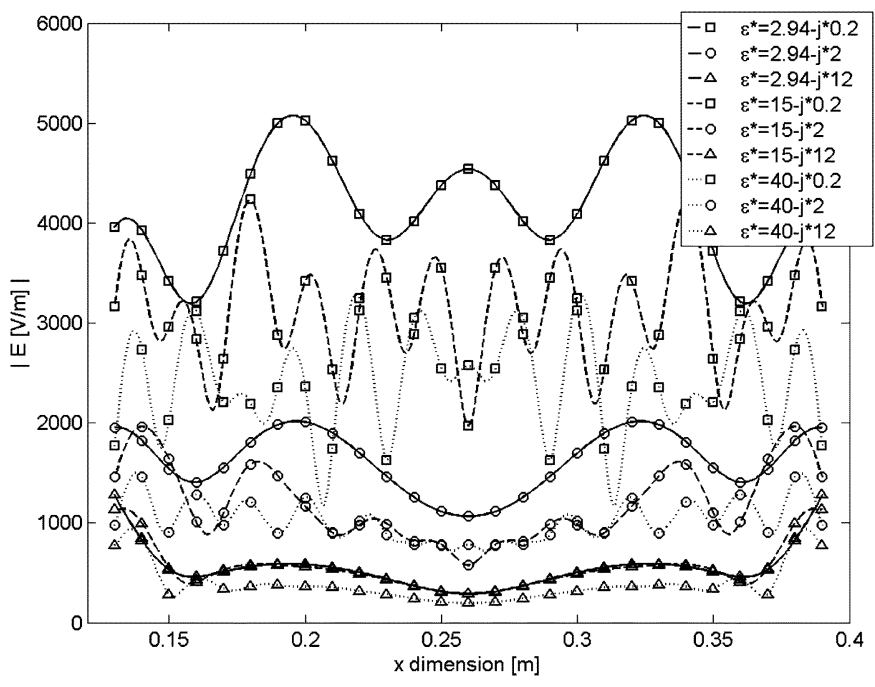

Fig. 8. Average electric-field distributions at the material center as a function of the sample dielectric constant and loss factor for position III. $t=10 \mathrm{~mm}$.

to achieve field uniformity. Consequently, it appears that, for these kinds of dielectrics, it is highly recommendable to place the mode stirrers as close as possible to the sample. However, for medium- and high-loss dielectrics, the electric field follows a very similar pattern, which seems to indicate that the mode stirrers' arrangement of scenario I does not influence pattern uniformity so much as it does for low-loss materials.

We can also appreciate from Fig. 7 the different electric-field intensities for the three materials regardless of the stirrer position. In order to identify the main cause for these differences in magnitude, a combination of dielectric constant and loss factor of the considered materials has been simulated for Position III of the stirrer, and the comparison is showed in Fig. 8.

From Fig. 8, the first effect that can be clearly appreciated is a low electric-field intensity for high-loss materials $\left(\varepsilon^{\prime \prime}=12\right)$ and, once again, a high concentration of this field at the edges, avoiding the penetration of fields within the sample volume. This low value is associated to the poor coupling at the air-material interface and it is almost independent of the sample dielectric constant. 
TABLE II

PARAMETERS AND GEOMETRY USED FOR FIGS. 9-11

\begin{tabular}{lll}
\hline Symbol & QuANTITY & Value \\
\hline$f$ & Frequency & $2.45 \mathrm{GHz}$ \\
$E_{\text {source }}$ & TE $_{10}$ Electric field peak value & $5 \times 10^{3} \mathrm{~V} / \mathrm{m}$ \\
$\varepsilon^{\prime}$ & Sample dielectric constant & $2.94,15,40$ \\
$\varepsilon^{\prime}$, & Sample loss factor & $0.2,2,12$ \\
$x_{c i}$ & Stirrer $1 x$ position (top) & $0.13 \mathrm{~m}$ \\
& Stirrer $2 x$ position (top) & $0.39 \mathrm{~m}$ \\
$L_{\text {stirrer }}$ & Stirrer $y$ sheet dimension & $0.1 \mathrm{~m}, 0.2 \mathrm{~m}, 0.3 \mathrm{~m}$ \\
$a$ & Stirrer angle (for each stirrer & $-48^{\circ}$ to $48^{\circ} ;-22^{\circ}$ to $22^{\circ} ;$ \\
& length, respectively) & $-14^{\circ}$ to $14^{\circ} ; 32 \mathrm{steps}$ \\
$L_{\text {sample }}$ & Sample Length & $0.26 \mathrm{~m}, \mathrm{x} \in[0.13,0.39] \mathrm{m}$ \\
$t$ & Sample thickness & $10 \mathrm{~mm}$ \\
& & $y_{\text {in }}=0.03 \mathrm{~m}$ \\
& & $y_{\text {end }}=0.04 \mathrm{~m}$ \\
& Multimode cavity dimensions & $x=0.52 \mathrm{~m}, y=0.54 \mathrm{~m}$ \\
\hline
\end{tabular}

Regarding other permittivity values, the main effect observed in Fig. 8 is a variable coupling depending upon the complex permittivity (real and imaginary parts) and the number of oscillations according to the wavelength inside the material, wherein lower loss factors allow the field to easily penetrate the sample and a smoother distribution can be achieved. For low dielectric constant values, the coupling is dominated by the loss factor and, for the other values, both magnitudes $\left(\varepsilon^{\prime}, \varepsilon^{\prime \prime}\right)$ have influence on the damping at the air-dielectric interface.

2) Scenario II: Vertical Stirrers With Angular Movement: For the geometry of the mode stirrer's configuration illustrated in Fig. 1(b), three lengths for the symmetric metallic blades are evaluated, called Lengths I-III, III being longer than the rest. The geometrical description and simulation parameters are given in Table II.

Fig. 9 shows the electric-field distribution in the multimode cavity for four different angles of the metallic blades with length III. We can appreciate from Fig. 9 that the blade movements provide very different electric-field spatial distributions in both the cavity and sample in a similar manner than with other stirrers' configurations.

Fig. 10 represents the computed average electric-field distribution at the center of the material samples with low, medium, and high losses for the three lengths of the stirrer blades.

When the permittivity is low (both dielectric constant and loss factor), the electric-field distribution is very different depending upon the stirrer length, and also very different from those shown in Fig. 7. Moreover, the field uniformity is better for the stirrer with length III, which confirms that the electric-field distribution uniformity is improved for those stirrer arrangements closer to the material. Once more, it is demonstrated that the stirrer design is extremely important to obtain a uniform electric power pattern.

For medium- and high-loss dielectrics, the electric-field distribution at the sample follows a very similar pattern than for the other stirrers' scenarios. Certainly, all the configurations showed higher electric-field intensities at the edges of the sample regardless of the mode-stirrer's configuration. This allows concluding that the electric-field distribution uniformity depends much more strongly on its geometry and permittivity than on

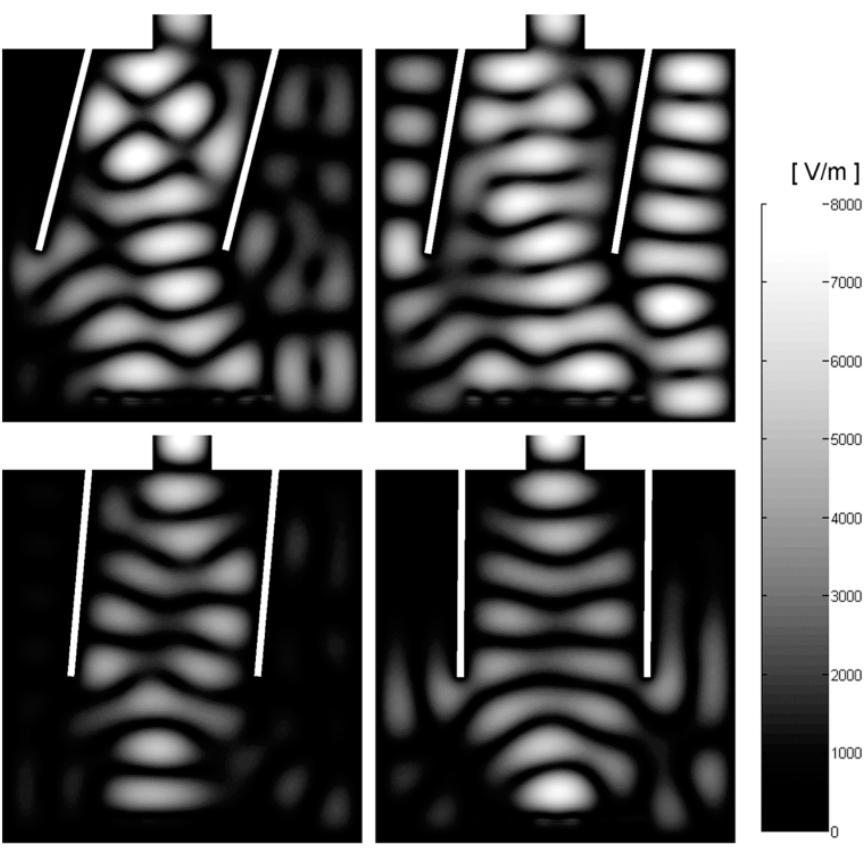

Fig. 9. Electric-field distribution (absolute value) in the multimode cavity for four different mode-stirrer angles $\left(w=-14^{\circ},-10^{\circ},-5^{\circ}\right.$, and $\left.-1^{\circ}\right) . \varepsilon^{\prime}=$ $40, \varepsilon^{\prime \prime}=12$, and $t=10 \mathrm{~mm}$

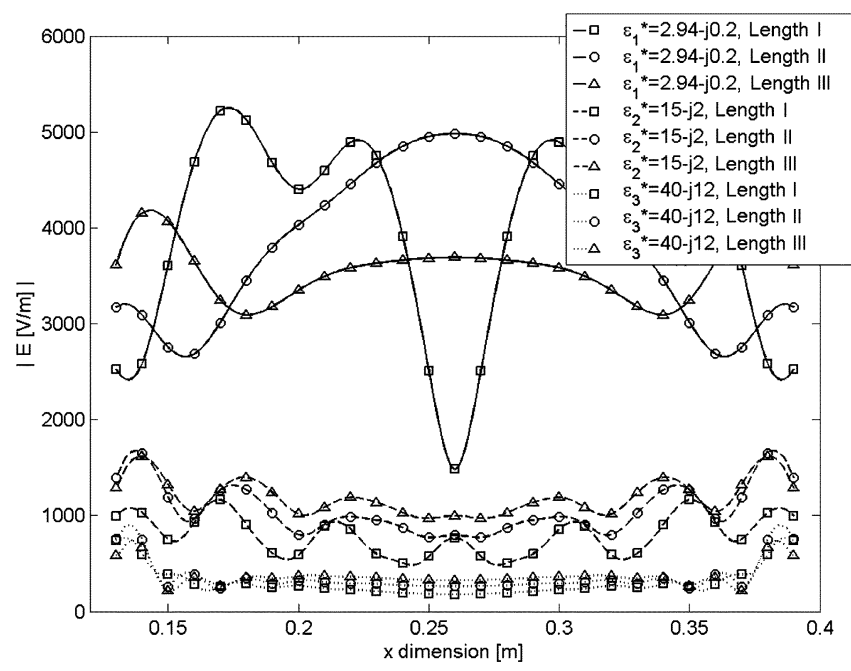

Fig. 10. Average electric-field distributions in the material in the microwave applicator as a function of the sample permittivity and angular stirrer length. $t=10 \mathrm{~mm}$.

the stirrer configuration. Thus, for these kinds of materials, new strategies must be searched in order to achieve the desired electric-field uniformity and thus, avoid, for instance, runaway effects [2].

Fig. 11 shows the average electric-field distribution calculated for length III of the blade stirrer, applied to a combination of dielectric constant and loss factor values of the materials mentioned above. The same conclusions derived from Fig. 8 apply here with this new stirrer's scenario.

\section{B. GPWA}

The electric-field distribution in the multimode microwave applicator seems to be independent of the stirrer arrangement for medium and high dielectric loss materials, and the electric- 


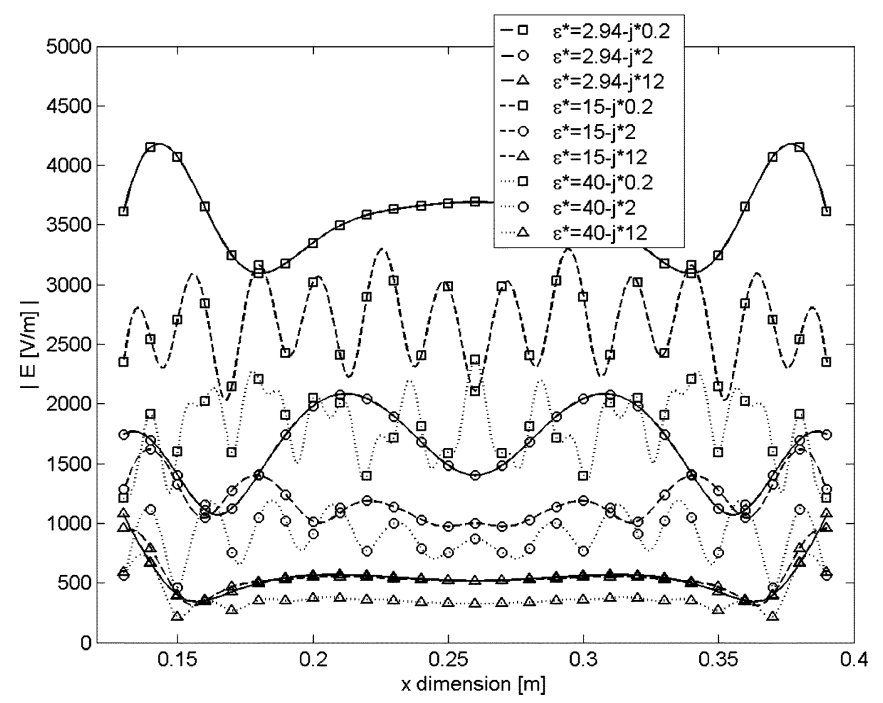

Fig. 11. Average electric-field distributions at the material center as a function of the sample dielectric constant and loss factor for length III. $t=10 \mathrm{~mm}$.

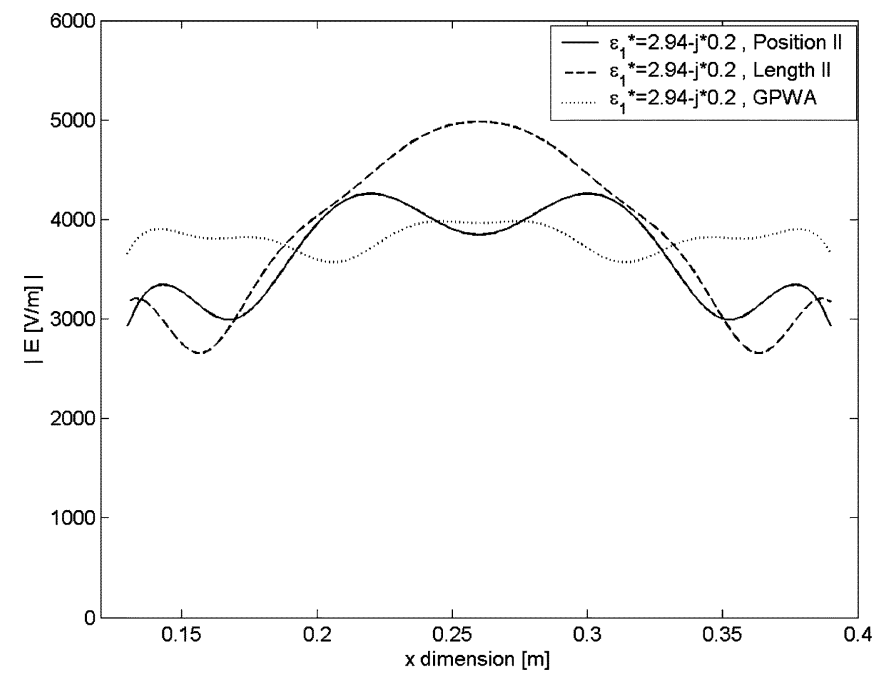

Fig. 12. Comparison of the electric-field distribution given by the FEM approach and by the GPWA for several stirrer's arrangements. $t=10 \mathrm{~mm}$.

field behavior is very similar to that provided by plane waves propagating in lossy media. Due to this, here we compare two different computing approaches for these kinds of applicators: the first approach is given by the FEM procedure described in [1], while the second is the one that uses the generalized planewave equations. The GPWA is used to obtain the electric-field distribution within the sample and the simulated patterns are compared to that obtained for the different stirrers' schemes and dielectric materials.

Figs. 12 and 13 show several electric-field distributions at the center of the sample for different dielectric permittivities and stirrers' configurations calculated in previous sections and comparing those obtained with the GPWA.

It is clearly observed in Fig. 13 that the electric-field distribution computed through the GPWA is very similar to that obtained for all that stirrers' arrangements when considering medium- and high-loss dielectric materials. This implies that,

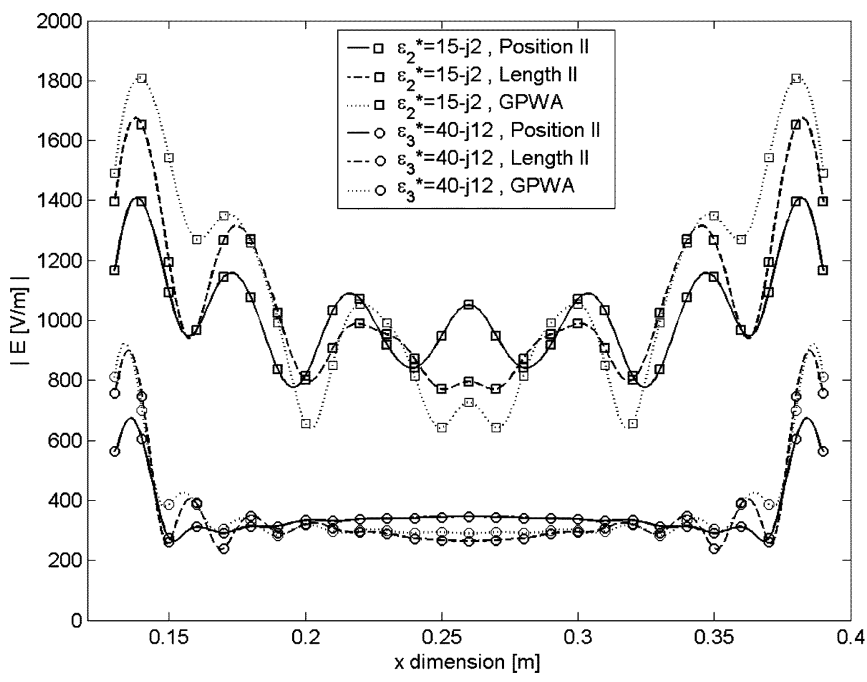

Fig. 13. Comparison of the electric-field distribution given by the FEM approach and by the GPWA for several permittivities and stirrer's arrangements. $t=10 \mathrm{~mm}$.

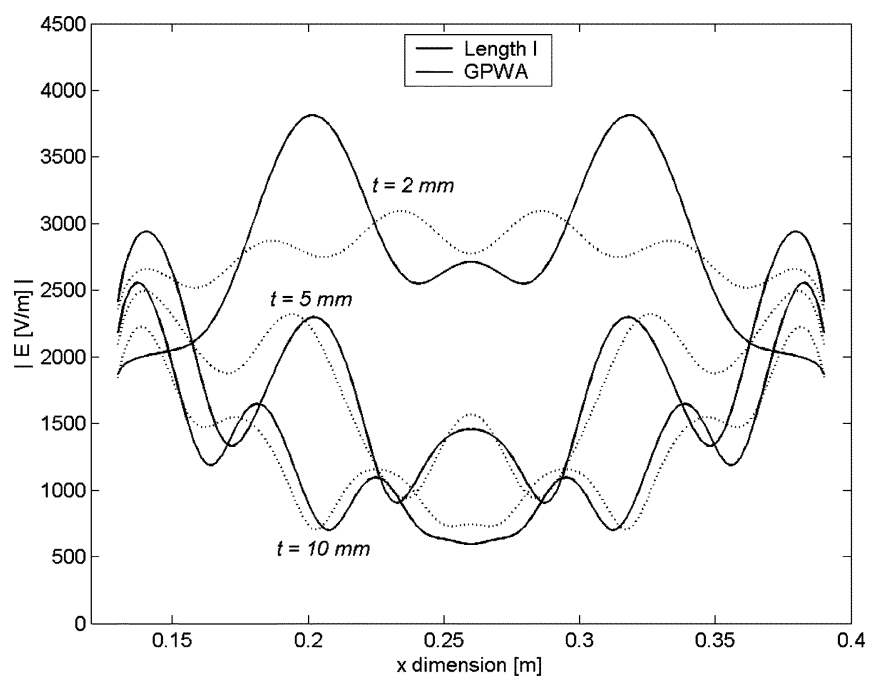

Fig. 14. Comparison of the electric-field distribution given by the FEM approach and by the GPWA for angular stirrer length I and several dielectric thicknesses. $\varepsilon^{*}=15-j 2$.

for these kinds of materials, the procedure summarized in Section II-A and used to compute the electric-field distribution within the dielectric sample can be avoided by the use of the GPWA, which is a much more simple and efficient technique in terms of computational cost. In fact, while the GPWA implies only one simulation and a very reduced computational domain only limited to the sample size, the method presented in [1] requires 32 simulations to obtain an acceptable precision. Unfortunately, Fig. 12 also shows that, for low-loss materials, the electric-field distributions cannot be acceptably predicted by the GPWA. In fact, for these kinds of dielectrics, the stirrer configuration very strongly determines the electric-field deposition within the sample.

On the other hand, Fig. 14 shows the range of application for GPWA versus the sample thickness. In this case, the relative 
complex permittivity is fixed at $\varepsilon_{r 2}=15-j 2$, while the electric-field pattern is computed for three different sample thicknesses $(t=2,5$, and $10 \mathrm{~mm})$ and the angular stirrer in Length $\mathrm{I}$.

The electric-field patterns computed through the GPWA are compared this time to the stirrers' configuration described in scenario I. Results indicate that the GPWA can predict the electric-field patterns for thick materials better than for thin samples. Moreover, for $t=2 \mathrm{~mm}$, the GPWA electric-field prediction strongly differs to the results given by the FEM procedure. However, for thicker materials, both methods provide very similar results.

These results indicate that, at least for thick dielectrics with moderate or high losses, the computation of the electric-field patterns in multimode microwave-heating cavities that contain stirrers can be solved through a very simple theory based on plane-wave propagation. This is explained by the fact that the TE and TM modes excited by the mode stirrers' movement create average electric-field distributions that can be associated to plane waves.

\section{CONCLUSIONS}

In this paper, a comparison of the performance of different mode stirrers' configurations has been carried out for low-, medium-, and high-loss dielectric materials. Simulated results have shown that both the sample permittivity and stirrer configuration have a strong influence on the field spatial distribution. Similar electric-field patterns have been obtained for all the evaluated stirrer scenarios, mainly for medium- and high-loss dielectrics. For these materials, no stirrer configuration has been able to avoid edge overheating. However, for low-permittivity materials, the stirrers' design seems to be very critical in order to obtain an acceptable uniform electric-field distribution within the sample. Stirrer designs with movements nearer the material have shown an important improvement in the uniformity in all simulated scenarios.

Additionally, the computation of the electric-field distribution through the GPWA has been demonstrated to be very similar to that obtained with the FEM approach for medium- and high-loss samples, but different for low-loss dielectrics. This indicates that, for thick high-loss materials, the stationary waves, induced by the reflections of the dielectric-air interface, determine the electric-field distribution, leaving the influence of the stirrers' configuration to a minor role. For low-loss materials, however, the role of stirrer's arrangements becomes of the utmost importance. Hence, the main effect of stirrers on high-loss materials is to generate different contributions that can be considered and modeled as equivalent plane waves on the dielectric. In this way, further research is envisaged to look for additional strategies that can reduce the overheating of the dielectric edges in high- and medium-loss materials.

\section{REFERENCES}

[1] P. Plaza-González, J. Monzó-Cabrera, J. M. Catalá-Civera, and D. Sánchez-Hernández, "A new approach for the prediction of the electric field distribution in multimode microwave-heating applicators with mode stirrers," IEEE Trans. Magn., vol. 40, no. 5, pp. 1672-1678, May 2004.
[2] A. C. Metaxas and R. J. Meredith, Industrial Microwave Heating. London, U.K.: Peter Peregrinus, 1983, pp. 71-103.

[3] T. V. Chow-Ting-Chan and H. C. Reader, Understanding Microwave heating Cavities. London, U.K.: Artech House, 2000, pp. 126-163.

[4] L. Zhou, V. M. Puri, and R. C. Anantheswaran, "Effect of temperature gradient on moisture migration during microwave heating," Dry. Technol., vol. 12, pp. 777-798, 1994.

[5] P. Chen and P. S. Schmidt, "An integral model for dielectrically-enhanced dying of hygroscopic and nonhygroscopic materials," Dry. Technol., vol. 8, pp. 907-930, 1994.

[6] C. A. Balanis, Advance Engineering Electromagnetics. New York: Wiley, 1989, pp. 352-470.

[7] C. Johnson, Numerical Solution of Partial Differential Equations by the Finite Element Method. Lund, Sweden: Studentlitteratur, 1987.

[8] C. Johnson and K. Eriksson, "Adaptive finite element methods for parabolic problems I: A linear model problem," SIAM J. Numer. Anal., vol. 28, pp. 43-77, 1991.

[9] R. F. Harrington, Field Computation by Moment Methods. Piscataway, NJ: IEEE Press, 1993.

[10] P. Kopyt and M. Celuch-Marcysiak, "FDTD modeling and experimental verification of electromagnetic power dissipated in domestic microwave ovens," J. Telecommun. Inf. Technol., vol. 1, pp. 59-65, 2003.

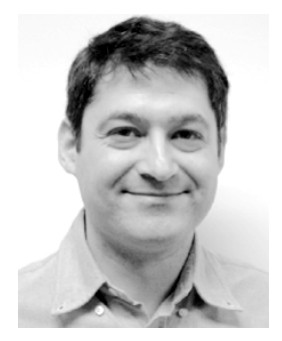

Pedro Plaza-González was born in Elda (Alicante), Spain, on June 1971. He received the Dipl. Ing. degree in telecommunications engineering from the Universidad Politécnica de Valencia, Valencia, Spain, in 1998, and is currently working toward the Ph.D. degree at the Universidad Politécnica de Valencia.

Since 1999, he has been a Research Assistant with the Microwave Heating Group (GCM), Universidad Politécnica de Valencia. His current research areas are focused on microwave-assisted heating and drying processes, microwave power instrumentation, and temperature control in microwave power systems.

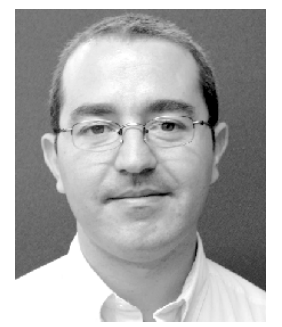

Juan Monzó-Cabrera was born in Elda (Alicante), Spain, on January 1973. He received the Dipl. Ing. and Ph.D. degrees in telecommunications engineering from the Universidad Politécnica de Valencia, Valencia, Spain, in 1998 and 2002, respectively.

From 1999 to 2000, he was a Research Assistant with the Microwave Heating Group (GCM), Universidad Politécnica de Valencia. In 2000, he joined the Departamento de Teoría de la Señal y Radiocomunicaciones, Universidad Politécnica de Cartagena, Cartagena, Spain, as an Associate Lecturer. He is currently an Associate Lecturer with the Departamento de Tecnologías de la Información y Comunicaciones, Universidad Politécnica de Cartagena. He has coauthored over 30 papers in referred journals and conference proceedings. He holds one patent regarding microwave heating industrial processes. His current research areas are microwave-assisted heating and drying processes, microwave applicator design and optimization, and numerical techniques in electromagnetics. $\mathrm{He}$ is a reviewer of several international journals.

Dr. Monzó-Cabrera is a member of the Association of Microwave Power in Europe for Research and Education (AMPERE), a European-based organization devoted to the promotion of RF and microwave energy. 


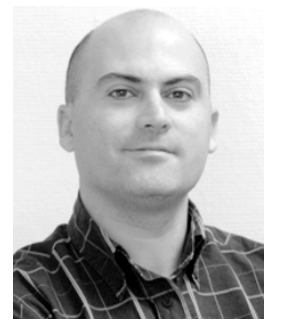

Jose M. Catalá-Civera (M'04) received the Dipl. Ing. and Ph.D. degrees in telecommunications engineering from the Universidad Politécnica de Valencia, Valencia, Spain, in 1993 and 2000, respectively.

From 1993 to 1996, he was a Research Assistant with the Microwave Heating Group, Universidad Politécnica de Valencia, where he was involved with microwave equipment design for industrial applications. Since 1996, he has been with the Departamento de Comunicaciones, Universidad Politécnica de Valencia, where he received the Readership in 2000. He is currently Head of the Microwave Applications Research Group, Advance Communications and Information Technologies Research Institute (ITACA), Universidad Politécnica de Valencia. He has coauthored approximately 60 papers in referred journals and conference proceedings and over 50 engineering reports for companies. $\mathrm{He}$ holds five patents. His research interests are the design and application of microwave theory and applications, the use of microwaves for electromagnetic heating, microwave cavities and resonators, measurement of dielectric and magnetic properties of materials, and development of microwave sensors for nondestructive testing. He is reviewer of several international journals.

Dr. Catala-Civera is member of the International Microwave Power Institute (IMPI). He is currently a Board member of the Association of Microwave Power in Europe for Research and Education (AMPERE), a European-based organization devoted to the promotion of RF and microwave energy.

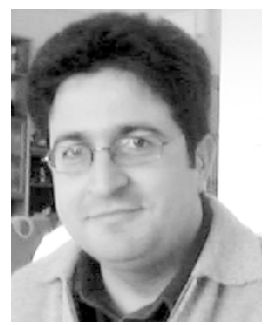

David Sánchez-Hernández (M'00) received the Dipl.-Ing. in telecommunications engineering from the Universidad Politécnica de Valencia, Valencia, Spain, in 1992, and the Ph.D. degree from King's College, University of London, London, U.K., in 1996.

From 1992 to 1994, he was a Research Associate with The British Council-CAM, King's College London, where he was involved with active and dual-band microstrip patch antennas. In 1994, he became a European Union (EU) Research Fellow with King's College London, where he was involved with several joint projects at 18,38 , and $60 \mathrm{GHz}$ related to printed and integrated antennas on GaAs, microstrip antenna arrays, sectorization, and diversity. In 1997, he rejoined the Universidad Politécnica de Valencia, where was Co-Leader of the Antennas, Microwaves and Radar Research Group and the Microwave Heating Group. In early 1999, he received the Readership from the Universidad Politécnica de Cartagena, and was appointed Vice Dean of the School for Telecommunications Engineering and Leader of the Microwave, Radiocommunications and Electromagnetism Engineering Research Group. In late 1999, he became Vice Chancellor for Innovation and Technology Transfer with the Universidad Politécnica de Cartagena, and a member of several foundations and societies for promotion of research and development in the autonomous region of Murcia, Spain. In May 2001, he became the Official Advisor in Technology Transfer and a member of The Industrial Advisory Council of the Autonomous Government of the Region of Murcia, Spain. In July 2003, he became Head of the department. He has authored or coauthored over 30 scientific journal papers and over 70 conference contributions. He is a reviewer of several international journals and conferences. He holds five patents. His current research interests encompass all aspects of the design and application of printed and integrated antennas and monolithic microwave integrated circuits (MMICs) for mobile communications, microwave heating, electromagnetic dosimetry issues, and multiple-input-multiple-output (MIMO) systems for wireless communications.

Dr. Sánchez-Hernández is an Institution of Electrical Engineers (IEE) Chartered Engineer (Stage 1). He is a member of the IEE Ampere Board member, the European Committee for Electrotechnical Standardization (CENELEC) through the Technical Committee TC106X (Electromagnetic Fields in the Human Environment), and the IEE RF and Microwave Circuits and Systems Professional Network Board. He was the recipient of the Research and Development J. Langham Thompson Premium presented by the Institution of Electrical Engineers, as well as other Spanish national research and development tutored awards. 